\title{
High Flux Isotope Reactor Quarterly Report April, May, and June of 1976
}

\author{
R. V. McCord \\ B. L. Corbett
}




\section{DISCLAIMER}

This report was prepared as an account of work sponsored by an agency of the United States Government. Neither the United States Government nor any agency Thereof, nor any of their employees, makes any warranty, express or implied, or assumes any legal liability or responsibility for the accuracy, completeness, or usefulness of any information, apparatus, product, or process disclosed, or represents that its use would not infringe privately owned rights. Reference herein to any specific commercial product, process, or service by trade name, trademark, manufacturer, or otherwise does not necessarily constitute or imply its endorsement, recommendation, or favoring by the United States Government or any agency thereof. The views and opinions of authors expressed herein do not necessarily state or reflect those of the United States Government or any agency thereof. 


\section{DISCLAIMER}

Portions of this document may be illegible in electronic image products. Images are produced from the best available original document. 
Printed in the United States of America. Available from National Technical Information Service

U.S. Department of Commerce

5285 Port Royal Road, Springfield, Virginia 22161

Prire. Printed Copy $\$ 3.50$; Micuuliche $\$ 2.25$

This report was prepared as an account of work sponsored by the United States Government. Neither the United States nor the Energy Research and Development Administration/United States Nuclear Regulatory Commission, nor any of their employees, nor any of their contractors, subcontractors, or their employees, makes any warranty, express or implied, or assumes any legal liability or responsibility for the accuracy, completeness nr usefulneg of any infuillation, apparatus, product or process disclosed, or represents that its use would not infringe privately owned rights. 
Contract No. W-7405-eng-26

Operations Division

HIGH FLUX ISOTOPE REACTOR QUARTERLY REPORT

APRIL, MAY, AND JUNE OF 1976

R. V. McCord

B. L. Corbett

Date Published: November 1976

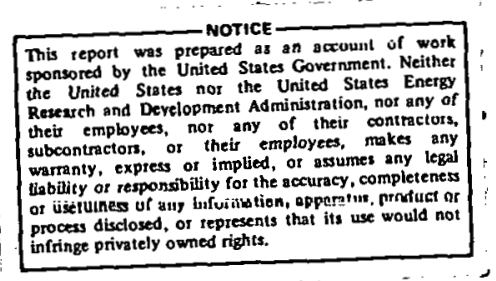

NOTICE This document contains information of a preliminary nature and was prepared primarlly for internal use at the Oak Ridge National Laboratory. It is subject to revision or correction and therefore does not represent a final report.

OAK RIDGE NATIONAL LABORATORY

Oak Ridge, Tennessee 37830

operated by

UNION CARBIDE CORPORATION

for the

ENERGY RESEARCH AND DEVELOPMENT ADMINISTRATION 


\section{THIS PAGE}

\section{WAS INTENTIONALLY \\ LEFT BLANK}


CONTENTS

$\underline{\text { Page }}$

SUMMARY . . . . ....................... . . . 1

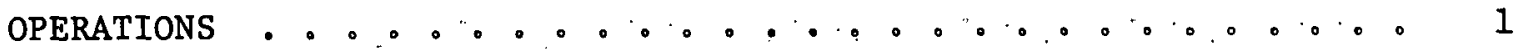

SHUTDOWNS .............................. 2

OPERATIONAL NOTES AND SPECIAL TESTS ............... 3

Reactor Bay In-Leakage. . . .............. 3

PLANT MAINTENANCE .....................。 4

INSTRUMENTATION AND CONTROLS . . ............... 4

SYSTEM SURVEILLANCE TESTS AND RESULTS . . . . . . . . . . 。

Vessel Head Studs ........ ................ 7

Stack Filters . . . . . . . . . . . . . . . . . . . . 7

REACTOR EXPERIMENTS ....................... 9

Experimental Facilities . . . . . . . . ......... 9

HFIR Target Loading . . . . . . . : . . . . . . . .... 9

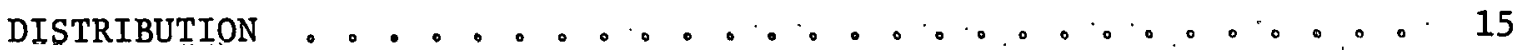




\section{SUMMARY}

Despite four end-of-cycle shutdowns this quarter, the reactor operated $95.6 \%$ of the time. This increased the on-stream time for the year to $95.3 \%$. A test of the reactor bay in-leakage determined that an exhaust flow rate of $3,050 \mathrm{cfm}$ is required to maintain a vacuum of $0.1 \mathrm{in}$ of water in this area.

\section{OPERATIONS}

Basic operating data for the quarter are listed in Table 1.

Table 1. HFIR Basic Operating Data

(April 1, 1976 through June 30, 1976)

\begin{tabular}{llll}
\hline & $\begin{array}{c}\text { This } \\
\text { Quarter }\end{array}$ & $\begin{array}{c}\text { Last } \\
\text { Quarter }\end{array}$ & $\begin{array}{c}\text { Year } \\
\text { To Date }\end{array}$ \\
\hline Total energy, MWd & 8,612 & 8,636 & 17,248 \\
Average power, MW/operating hr & 99.0 & 99.8 & 99.4 \\
Time operating, \% & 95.6 & 95.0 & 95.3 \\
Reactor availability, \% & 96.3 & $\because 97.2$ & 96.8 \\
$\begin{array}{l}\text { Reactor water radioactivity, } \\
\text { c/min (av) }\end{array}$ & 344,000 & 222,000 & \\
Pool water radioactivity, & & & \\
$\frac{\mathrm{c} / \mathrm{m} 1 \mathrm{n}}{\mathrm{m} 1}$ (av) & 54 & 42 & \\
\hline
\end{tabular}

The starting and ending dates for cycles 130, 131, 132, and 133 are presented in Table 2.. Cycle 134 was still in progress at the end of the quarter and 1392 MWd had been accumulated. 
Table 2. Cycles of Operation

\begin{tabular}{cccc}
\hline Cycle No. & Date Started & Date Ended & $\begin{array}{l}\text { Accumulated } \\
\text { Power (INd) }\end{array}$ \\
\hline 130 & $3-14-76$ & $4-6-76$ & 2324 \\
131 & $4-7-76$ & $4-30-76$ & 2301 \\
132 & $5-1-76$ & $5-22-76$ & 2040 \\
133 & $5-24-76$ & $6-16-76$ & 2278 \\
131 & $6-17-76$ & In progress & 1392 \\
\hline
\end{tabular}

The status of the HFIR fuel and control-plate inventory is indicated in Table 3.

Table 3. HFIR Material Inventory

\begin{tabular}{lcc}
\hline \multicolumn{1}{c}{ Item } & This & Last \\
\hline Quarter & Quarter \\
New fuel elements placed in service & 4 & 3 \\
Spent fuel elements on hand & 48 & 47 \\
Spent fuel elements shipped & 11 & 12 \\
New sets of shim plates placied in service & 5 & 0 \\
New sets of shim plates available for use & 0 & 0 \\
\hline
\end{tabular}

SHUTDOWNS

A summary of the HFIR shutdowns for the quarter is presented in Table 4。. Table 5 gives further details of the shutdowns.

Table 4. Summary of HFIR Shutdowns.

\begin{tabular}{|c|c|c|c|c|}
\hline Type & Number & & & Downtime (hr) \\
\hline & Scheduled & . & & \\
\hline End of cycle & 4 & $\because \quad \quad \ddots$ & & 95.383 \\
\hline & Unscheduled & & - & . \\
\hline Spurious trip & 1 & & & 0.100 \\
\hline
\end{tabular}


Table 5. Description of HFIR Shutdowns

\begin{tabular}{|c|c|c|}
\hline Date & $\begin{array}{c}\text { Downt ime } \\
\text { (hr) }\end{array}$ & Remarks \\
\hline & & Scheduled \\
\hline $4-6-76$ & 21.500 & $\begin{array}{l}\text { Fuel cycle } 130 \text { was completed at } 11: 54 \mathrm{p} . \mathrm{m} \text {. A total } \\
\text { power generation of } 2324 \mathrm{MWd} \text {.was obtained on fuel } \\
\text { elements } 126-0 \text { and } 126-\mathrm{I} \text {. The shutdown was } \\
\text { extended } n 8 \text { hours to complete a number of small } \\
\text { maintenance items. }\end{array}$ \\
\hline $4-30-76$ & 22.500 & $\begin{array}{l}\text { Fuel cycle } 131 \text { was completed at } 11: 12 \text { p.m. A total } \\
\text { power generation of } 2301 \text { MWd was obtained on fuel } \\
\text { elements } 127-0 \text { and } 127-1 \text {. The shutdown.was } \\
\text { extended } 28 \text { hours to install an experiment. }\end{array}$ \\
\hline $5-22-76$ & 38.183 & $\begin{array}{l}\text { Fuel cycle } 132 \text { was terminated at } 8: 00 \text { a.m. A total } \\
\text { power generation of } 2040 \text { MWd was obtained on fuel } \\
\text { elements } 128-0 \text { and } 128-I_{0} \text {. The cycle was termi- } \\
\text { nated early, and the shutdown was extended for } \\
\text { ORNL Family Day activities. }\end{array}$ \\
\hline $6-16-76$ & 13.200 & $\begin{array}{l}\text { Fuel cycle } 133 \text { was completed at } 12: 40 \mathrm{p} . \mathrm{m}_{\circ} \text { A total } \\
\text { power generation of } 2278 \text { MWd was obtained on fuel } \\
\text { elements } 130-0 \text { and } 130-\mathrm{I}_{\text {. }} \text {. }\end{array}$ \\
\hline & & Unscheduled \\
\hline $5-12-76$ & 0.100 & $\begin{array}{l}\text { Safety plates No. } 2 \text { and No. } 4 \text { dropped while a "heat } \\
\text { power": test was being performed on the Channel. } \\
\text { No. } 3 \text { safety instrumentation. }\end{array}$ \\
\hline
\end{tabular}

OPERATIONAL NOTES AND SPECIAL TESTS

Reactor Bay In-Leakage

The HFIR Operating Safety Limits specify that during reactor operation the minimum exhaust rate from the reactor bay shall be sufficient to maintain in-leakage through all building openings but shall not be less than $6,000 \mathrm{cfm}$. Actually, a vacuum of. $\sim 0.3 \mathrm{in}$ of water and an exhaust flow rate of $~ 49,500 \mathrm{cfm}$ is maintained during reactor operation.

To get an estimate of the minimum exhaust flow rate required to maintain a negative pressure in this area, after the end of cycle 130 the exhaust flow from the reactor bay was slowly throttled until the negative pressure dropped to $0.1 \mathrm{in}$ of water. It was determined that an exhaust flow of only $3,050 \mathrm{cfm}$ was sufficient to maintain a vacuum of 0.1 in. of water in the reactor bay. 
PLANT MAINTENANCE

Maintenance and changes in the various process systems are listed in Table 6 。

INSTRUMENTATION AND CONTROLS

Maintenance and changes in the various instrumentation systems are 1isted in Table 7 . 
Table 6。'Process System - Maintenance and Changes

\begin{tabular}{|c|c|c|}
\hline Date & Component & Remarks \\
\hline \multicolumn{3}{|r|}{ Primary System: } \\
\hline $4-7-76$ & $\begin{array}{l}\text { Pressure } \cdot \text { balance } \\
\text { system }\end{array}$ & $\begin{array}{l}\text { A leaking seal was replaced on the pressure balance system for the inner } \\
\text { control cylinder. Oil was added to the reservoir, and the entire } \\
\text { hydraulic system was degassed. }\end{array}$ \\
\hline $5-27-76$ & Cleanup pump & $\begin{array}{l}\text { A new breaker was installed in the starting circuit of primary cleanup } \\
\text { pump PU-2A. }\end{array}$ \\
\hline \multicolumn{3}{|r|}{ Secondary System. } \\
\hline $4-7-76$ & Makeup valve & $\begin{array}{l}\text { A new } 3 \text {-in. potable water makeup valve was installed at the cooling tower. } \\
\text { The valve was removed in a few days because it was too small. }\end{array}$ \\
\hline $5-1-76$ & $\begin{array}{l}\text { Flow control } \\
\text { valve }\end{array}$ & $\begin{array}{l}\text { Secondary. flow control valve FCV- } 377 \mathrm{~A} \text { developed a sluggish control action. } \\
\text { The valve operator was found disengaged from the valve stem. }\end{array}$ \\
\hline $6-16-76$ & Makeup valve & $\begin{array}{c}\text { A new 4-in。 potable water makeup valve was installed at the cooling tower. } \\
\text { Miscellaneous }\end{array}$ \\
\hline $5-12-76$ & Air compressor & Inlet and exhaust valves were replaced in air compressor $C-1 B$. \\
\hline
\end{tabular}


Table 7. Instrumentation - Maintenance and Changes

\begin{tabular}{|c|c|c|}
\hline Date & Component & Remarks \\
\hline & & Safety System \\
\hline $5-25-76$ & $\begin{array}{l}\text { Rod release timer } \\
\text { circuit }\end{array}$ & $\begin{array}{l}\text { A pulse generator was added to the rod release timer circuit as } \\
\text { authorized by Reactor Instrument and Controls Design Change Memo- } \\
\text { randim No. } 87 \text {. }\end{array}$ \\
\hline $6-1-76$ & $\Delta \mathrm{T}$ subtractor & $\begin{array}{l}\text { During a safety system test the No. } 2 \text { chamel flux-to-flow ratio test } \\
\text { would not trip the channel because of a faulty } \Delta \mathrm{T} \text { subtractor. The } \\
\text { channel was manually tripped until the faulty component was found } \\
\text { and replaced. }\end{array}$ \\
\hline $6-21-76$ & $\begin{array}{l}\text { Temperature } \\
\text { transmitter }\end{array}$ & $\begin{array}{l}\text { An inlet temperature transmitter, which had been producing erratic } \\
\text { readings on Channel No. 1, was replaced. } \\
\text { Servo System }\end{array}$ \\
\hline $4-20-76$ & Servo motor & $\begin{array}{l}\text { The Channel No. } 3 . \text { servo motor was replaced because it was using an } \\
\text { excessive amcunt of current. } \\
\text { Misciellaneous }\end{array}$ \\
\hline $6-21-76$ & Computer & $\begin{array}{l}\text { The computer printouts in the control roon became inoperable. Repairs } \\
\text { have not been completed. }\end{array}$ \\
\hline
\end{tabular}


SYSTEM - SURVEILLANCE TESTS AND RESULTS

\section{Vessel Head Studs}

The accumulated number of tensioning cycles on the reactor vessel head studs is presented in Table 8. These studs were designed for a. fatigue life of 40 cycles loading due to tensioning of the bolts and 730 full-pressure (1000 psig) cycles. Installation of new reactor vessel head studs was completed in June, 1972. The numbers in Table 8 represent the maximum cycles to. which any new stud has been exposed。

Table 8. Vessel Head Stud-Tensioning Cycles

\begin{tabular}{lccc}
\hline & $\begin{array}{c}\text { This } \\
\text { Quarter }\end{array}$ & $\begin{array}{c}\text { Last } \\
\text { Quarter }\end{array}$ & $\begin{array}{c}\text { Total } \\
\text { To Date }\end{array}$ \\
\hline Head bolts tensioned & 0 & 0 & 6 \\
1500 psig & & 0 & 0 \\
950 psig & 0 & 3 & 7 \\
650 psig & 5 & 106 \\
\hline
\end{tabular}

\section{Stack Filters}

Stack filtering systems in the special building hot exhaust (SBHE) and hot off-gas (HOG) systems were tested for particulate removal efficiency. Results of the most recent tests are tabulated in Table.9. 
Table 9. Partlculate ard Iodine Removal Efficleacy

\begin{tabular}{|c|c|c|c|c|c|c|c|c|c|c|c|c|c|}
\hline \multirow[b]{3}{*}{ F1lter Bank } & \multicolumn{4}{|c|}{ Methy I Iodide } & \multicolumn{4}{|c|}{ Elemental Iodine } & \multirow{3}{*}{$\begin{array}{c}\text { Filter } \\
\text { Position } \\
\end{array}$} & \multicolumn{4}{|c|}{ Particulate Retention } \\
\hline & \multicolumn{2}{|c|}{ Last Test } & \multicolumn{2}{|c|}{ Previous Test } & \multicolumn{2}{|c|}{ - Last Test } & \multicolumn{2}{|c|}{ Previous Test } & & \multicolumn{2}{|c|}{ Last Test ${ }^{\circ}$} & \multicolumn{2}{|c|}{ Previous Test } \\
\hline & Date & Eff., \% & Date & Eff.2\% & Date & Eff., $\%$ & Date & Eff., $\%$ & & Date & Eff., $\%$ & Date & Eff., \\
\hline SBHE, we: $t$ & $5-4-76$ & 48 & $10-23-75$ & 75 & $3-1 \varepsilon-7 \epsilon$ & 99.96 & $9-8-75$ & $99.9 \mathrm{C}$ & South & $3-26-76$ & 99.980 & $9-26-75$ & 99.996 \\
\hline : & : & & & & & & & & North & $3-26-76$ & $99.000 \star$ & $9-26-75$ & 99.997 \\
\hline SBHE, center & $5-11-76$ & 80 & $10-29-75$ & 88 & $3-30-76$ & 99.98: & $9-11-75$ & $99.9^{7}$ & South & $3-26-76$ & 99.988 & $9-26-75$ & 99.995 \\
\hline & . & & & & 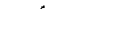 & & & & North & $3-26-76$ & 99.960 & $9-26-75$ & 99.958 \\
\hline SBHE, east & $5-6-76$ & 47 & $10-28-.5$ & 59 & $3-2 \geq-76$ & 99.97 & $10-9-75$ & $99.9 \mathrm{~L}$ & South & $3-26-76$ & 99.956 & $9-26-7 \dot{5}$ & 99.992 \\
\hline . & & - & & & & . & & ' & North & $3-26-76$ & $99.930 *$ & $9-26-75$ & 99.960 \\
\hline HOG, west & $5-13-76$ & 73 & $10-30-75$ & 90 & $4-6-75$ & 99.95 & $10-20-75$ & 99.93 & & · & . & & \\
\hline HOG, center & $5-18-76$ & 68 & $11-5-7.5$ & 44 & $4-22-75$ & $99.9 \mathrm{G}$ & $10-14-75$ & 99.99 & & & & & . \\
\hline HOG, east & $5-20-76$ & 98 & $i 1-4-75$ & 92 & $4-14-76$ & 99.90 & $10-15-75$ & 99.99 & & & $\cdot$ & + & \\
\hline
\end{tabular}

Filters were replaced. 


\section{REACTOR EXPERIMENTS}

Experimental Facilities

Usage of the various HFIR experiment facilities is tabulated in Table 10.

Table 10... Experiment Facility..Usage

\begin{tabular}{|c|c|c|}
\hline Facility & Description. & Division \\
\hline $\mathrm{RB}-1$ & Isotope production & Operations \\
\hline $\mathrm{RB}-2$ & Isotope production & Operations \\
\hline $\mathrm{RB}-3$ & Isotope production & Operations \\
\hline $\mathrm{RB}-4$ & HFIR tensile specimens & Operations \\
\hline $\mathrm{RB}-5$ & HTGR fuel irradiations & Reactor \\
\hline $\mathrm{RB}-6$ & Isotope production & Operations \\
\hline $\mathrm{RB}-7$ & HTGR fuel irradiațions & Reactor \\
\hline $\mathrm{RB}-8$ & Isotope production & Operations \\
\hline $\mathrm{CR}-1$ & Isotope production & Operations \\
\hline $\mathrm{CR}-2$ & Isotope production & Operations \\
\hline $\mathrm{CR}-3$ & Californium production. & Chemical Technology \\
\hline $\mathrm{CR}-4$ & Californium production & Chemical Technology \\
\hline $\mathrm{CR}-5$ & Isotope production & Operations \\
\hline $\mathrm{CR}-6$ & Isotope production & Operations \\
\hline $\mathrm{CR}-7$ & Isotope production & Operations \\
\hline $\mathrm{CR}-8$ & Isotope production & Operations \\
\hline VXF-1 & HFIR corrosion specimen & Operations \\
\hline VXF-7 & Pneumatic tube. & Analytical Chemistry \\
\hline $\mathrm{EF}-2$ & Noise analysis studies & $\begin{array}{l}\text { Instrumentation and Controls } \\
\text { and Operations }\end{array}$ \\
\hline $\mathrm{HB}-1$ & Neutron diffractometer & Solid State \\
\hline $\mathrm{HB}-2$ & Neutron diffractometer & Chemiotry \\
\hline $\mathrm{HB}-3$ & Neutron diffractometer & Solid State. \\
\hline $\mathrm{HB}-4$ & Neutron diffractometer & Solid State \\
\hline
\end{tabular}

HFIR Target Loading

A description of the HFIR target loading for each of the operating cycles this quarter is presented in Figures 1, 2, 3, 4, and 5 . 


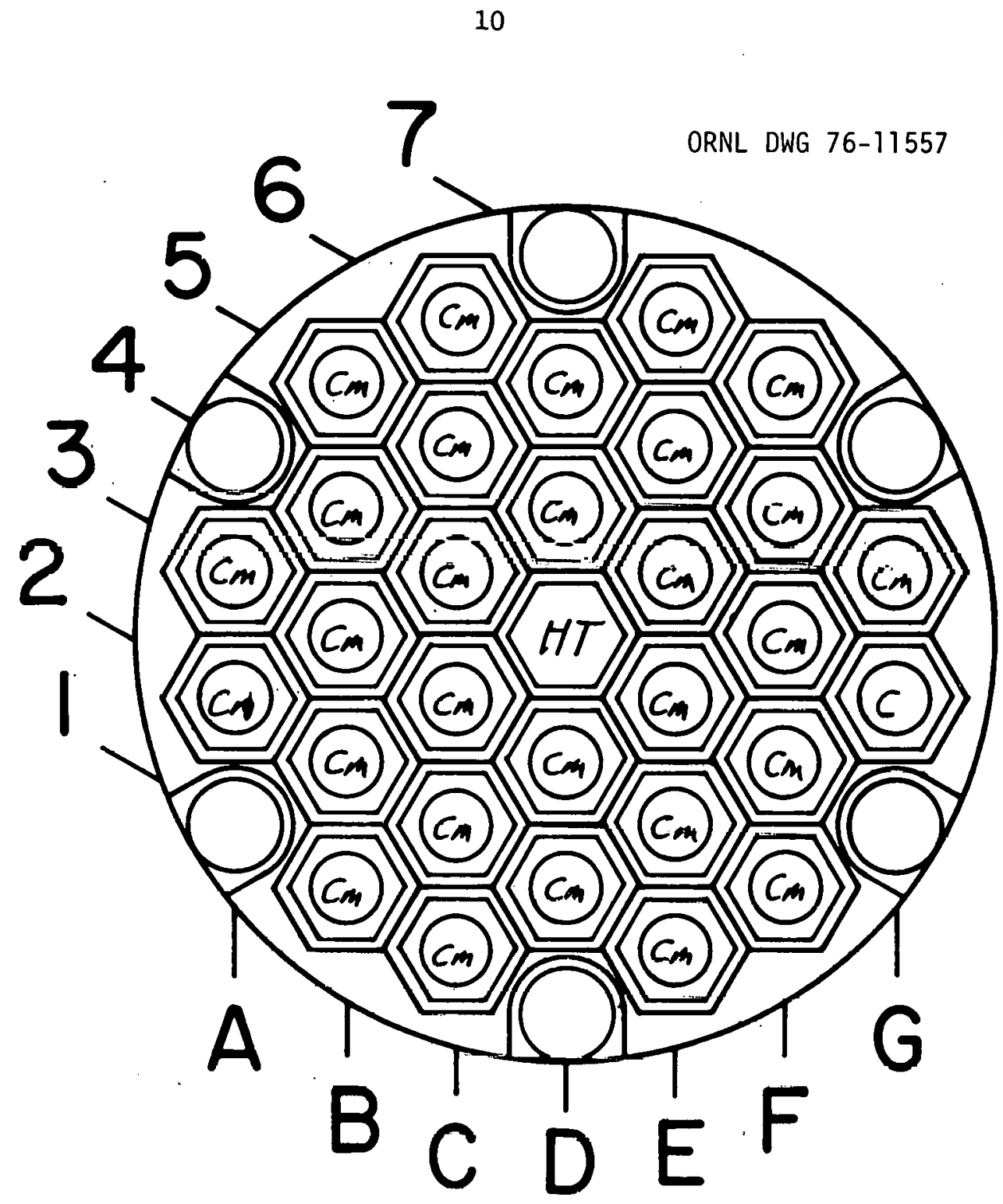

TARGET TYPE

NUMBER

PLUTONIUM (PU).

CURIUM (Cm)

STAINLESS STEEL (SST)

GRAPHITE (C)

ALUMINUM (AI)

HYDRAULIC TUBE (HT)

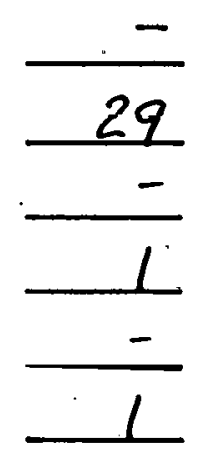

Fig. 1. HFIR Target Loading for Cycle 130 - March 14, 1976 


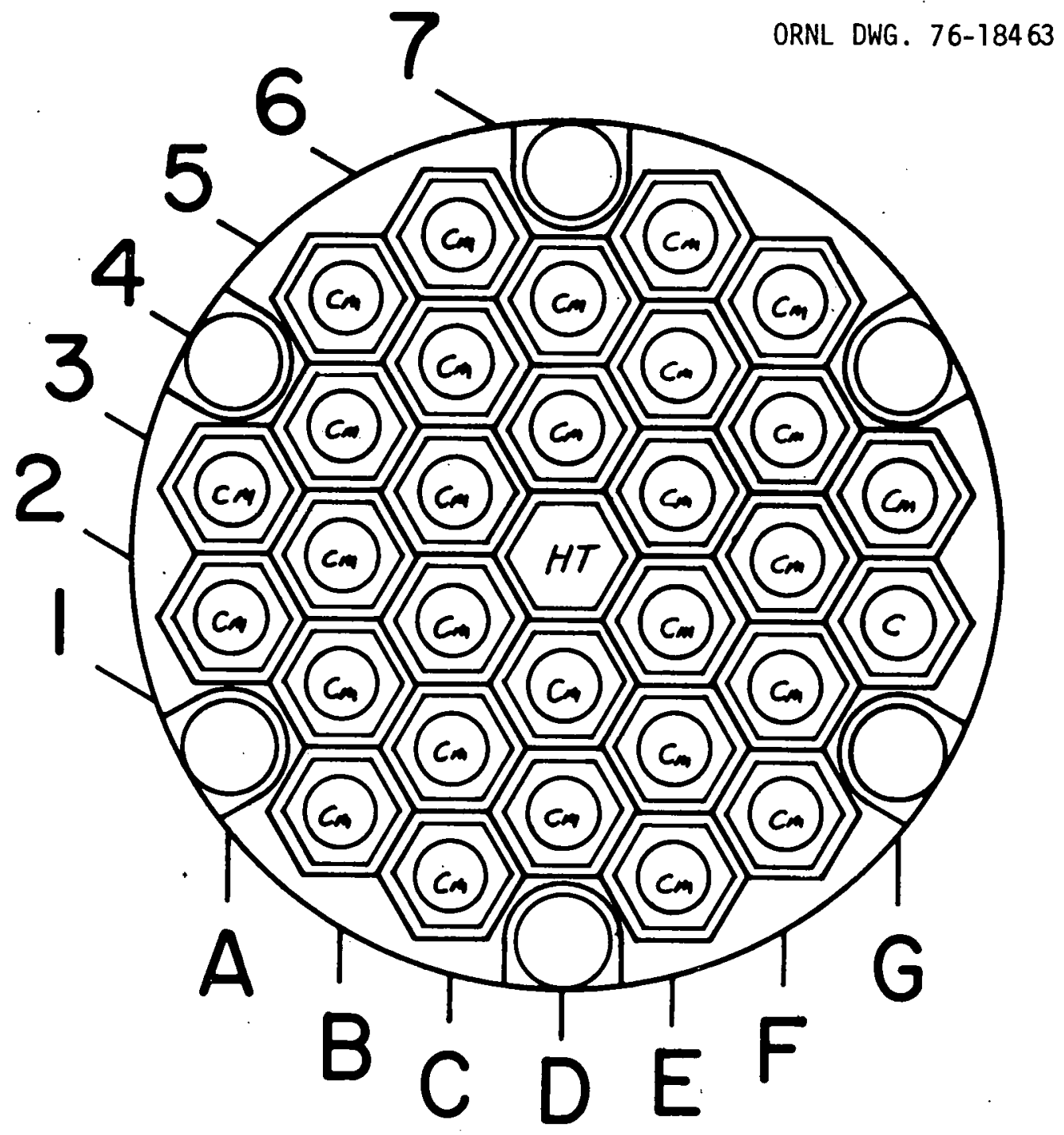

TARGET TYPE

NUMBER

PLUTONIUM (Pu)

CURIUM (Cm)

STAINLESS STEEL (SST)

GRAPHITE (C)

ALUMINUM (AI)

HYDRAULIC TUBE (HT)

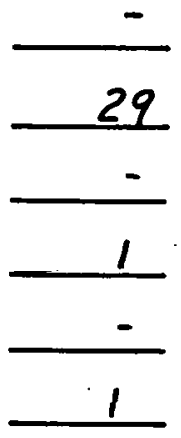

Fig. 2. HFIR Target Loading for Cycle 131 - Apri1 7, 1976 


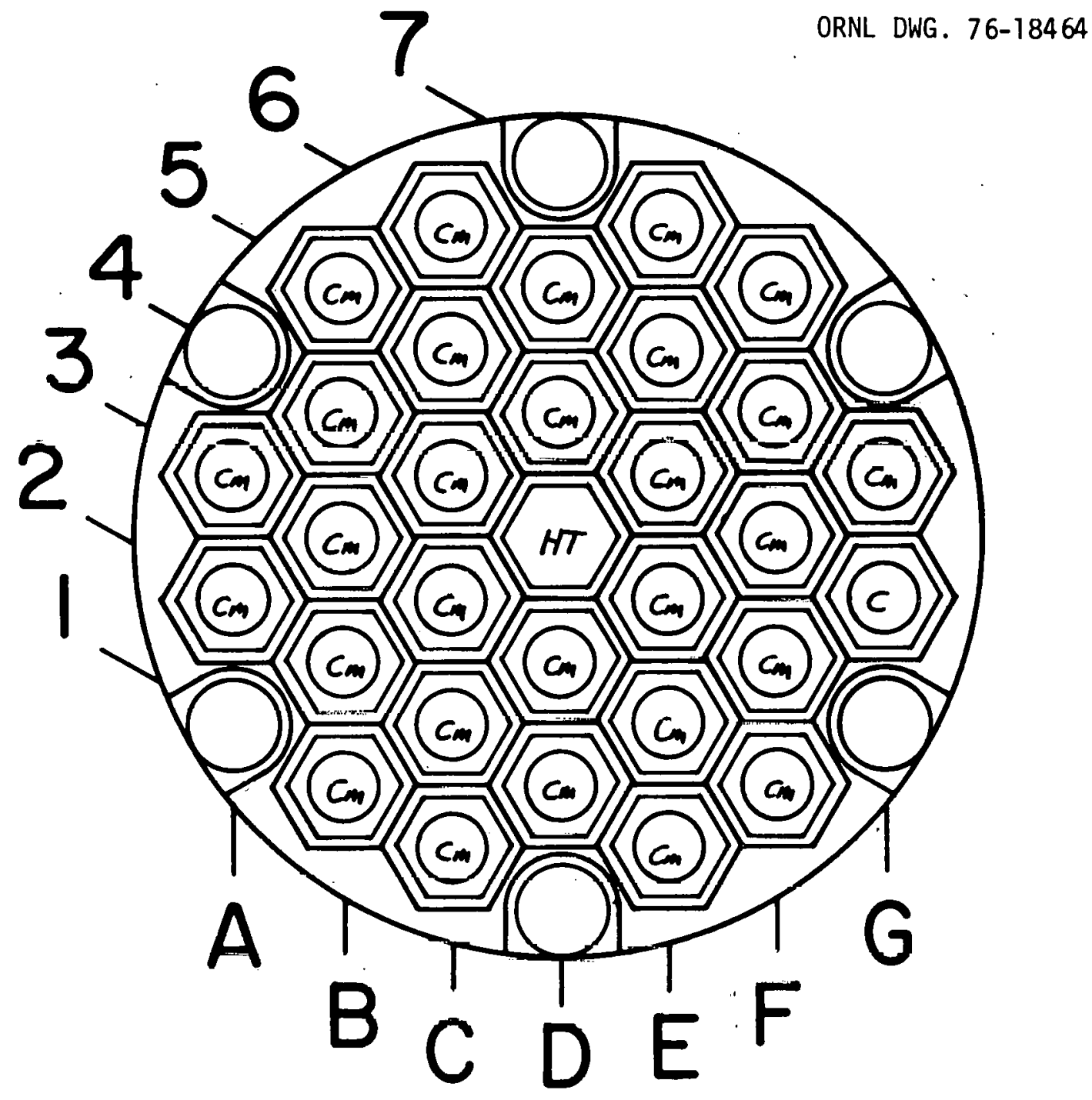

TARGET TYPE

PLUTONIUM (Pu)

CURIUM (Cm)

STAINLESS STEEL (SST)

GRAPHITE (C)

ALUMINUM (AI)

HYDRAULIC TUBE (HT)
NUMBER

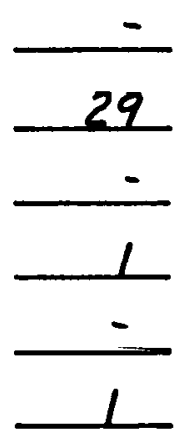

Fig. 3. HFIR Target Loading for Cycle 132 - May 1, 1976 


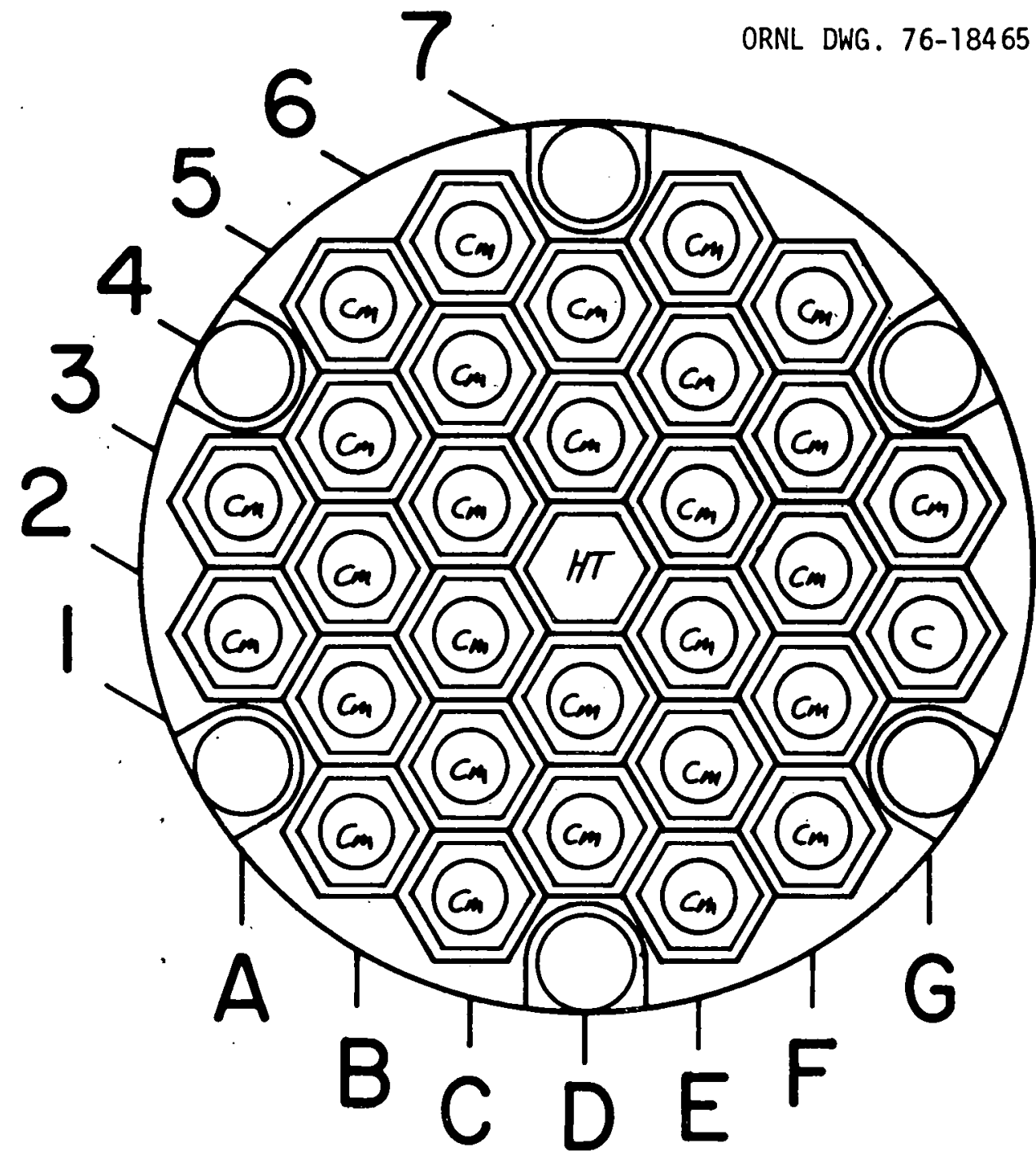

TARGET TYPE

NUMBER

PLUTONIUM (Pu)

CURIUM (Cm)

STAINLESS STEEL (SSTT)

GRAPHITE (C)

ALUMINUM (AI)

HYDRAULIC TUBE (HT)

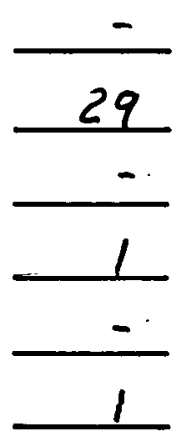

F1g. 4. HFIR Target Loading for Cycle 133 - May 23, 1976 


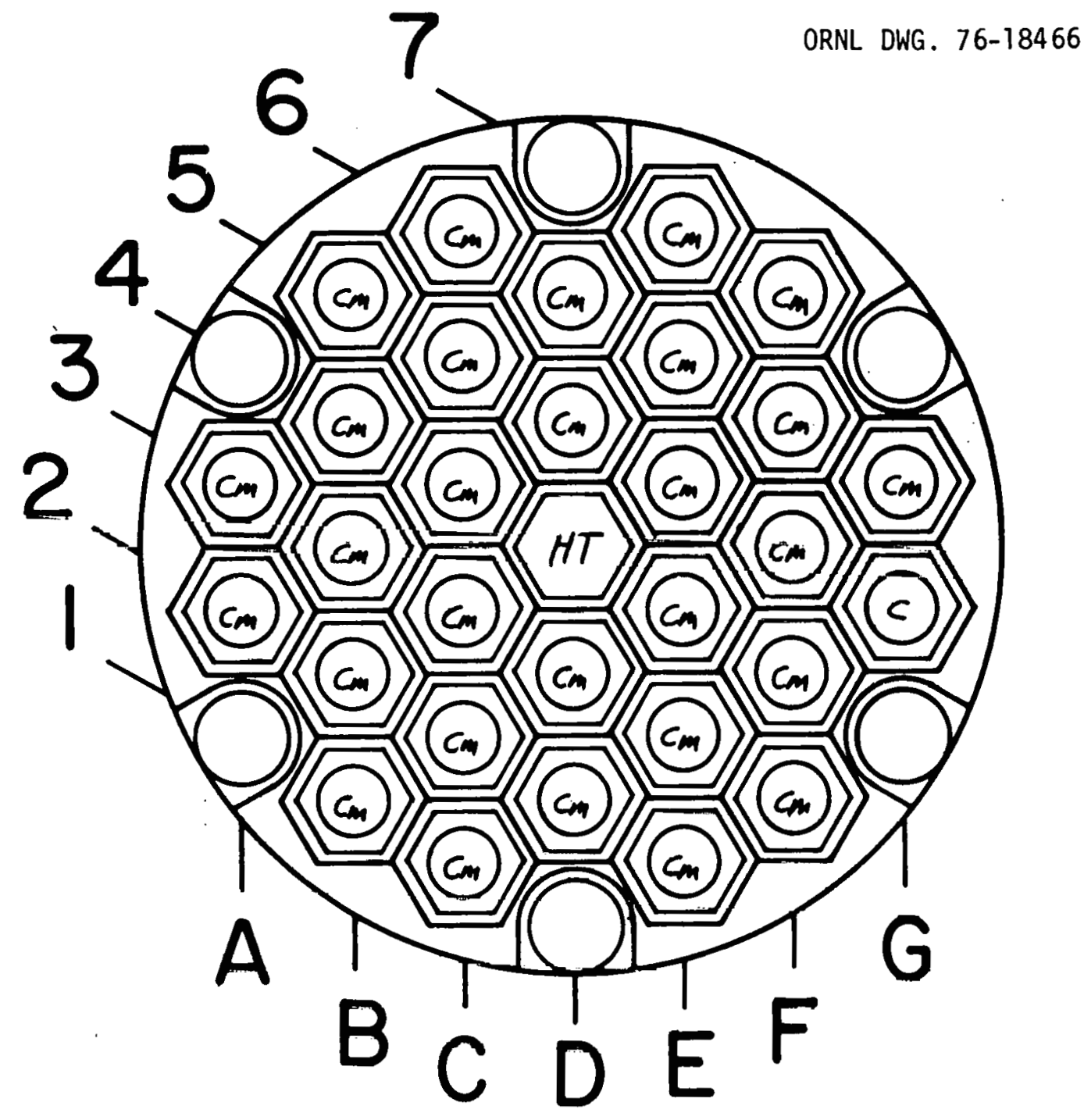

TARGET TYPE

PLUTONIUM (Pu)

CURIUM (Cm)

STAINLESS STEEL (SST)

GRAPHITE (C)

ALUMINUM (AI)

HYDRAULIC TUBE (HT)
NUMBER

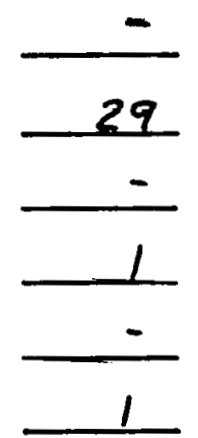

Fig. 5. HFIR Target Loading for Cycle 134 - June 17, 1976 
INTERNAL DISTRIBUTION

1. G. M. Adamson

2. R. G. Affel

3. J. L. Anderson

4. D. S. Asquith

5. K. S. Belitz

6. J. E. Bigelow

7. D. S. Billington

8. F. T. Binford

9. C. D. Cagle

10. W, R. Casto

11. R. D. Cheverton

12. T. E. Cole

13. J. A. Conlin

14-15. B. L. Corbett

16. J. A. Cox

17. W. H. Culbert

18. F. L. Culler

19. S. Jo Ditto

20. G. J. Dixon

21. K. Farrell

22. A. P. Fraas

23. E. D. Guptón

24. T. P. Hamrick

25. V. O. Haynes

26. C. H. Helton

27. S. S. Hurt, III

28. E. B. Johnson

29. S. I. Kaplan

30. 0. L. Keller

31. E. M. King
32. H. V. Klaus

33. R。 W. Knight

34. W. C. Koehler

35. E. Lamb

36. H. A. Levy

37. J. Lewin

38-43. R. V. McCord

44. J. R. McGuffey

45. H. A. McLain

46. J。 R. McWherter

47. Jo Mo Miller

48. L. C. Oakes

49. H. Postma

50. K. H. Poteet

51. M. E. Ramsey

52. R。 L。 Scott, Jr.

53. W. Ho Sides

54. T. M. Sims

55. M. Jo Skinner

56. J. H. Swanks

57. J. R. Thomas

58. D. B. Trauger

59. J. R。 Weir

60. K. W. West

61. M. Ko Wilkinson

62. E. Jo Witkowski

63-64. Central Research Library

65. Document Reference Section

66-67. Laboratory Records Department

68. Laboratory Records, ORNL R.C.

\section{EXTERNAL DISTRIBUTION}

69-95. 'lechnical Information Center, Oak Ridge

96-97. M. B. Biles, ERDA Washington

98. K. J. Bobin, Belgium

99. J. L. Burnett, ERDA Washington

100. H. N. Culver, ERDA

101. M. Jacquemain, France

102. G. L. Rogosa, ERDA Washington

103. Research and Technical Support Division, ORO 